\title{
Hierarchical Discrete Relaxation
}

\author{
Richard C. Wilson and Edwin R. Hancock \\ Department of Computer Science, University of York \\ York, Y01 5DD, UK
}

\begin{abstract}
Our aim in this paper is to develop a Bayesian framework for matching hierarchical relational models. Such models are widespread in computer vision. The framework that we adopt for this study is provided by iterative discrete relaxation. Here the aim is to assign the discrete matches so as to optimise a global cost function that draws information concerning the consistency of match from different levels of the hierarchy. Our Bayesian development naturally distinguishes between intra-level and inter-level constraints. This allows the impact of reassigning a match to be assessed not only at its own (or peer) level of representation, but also upon its parents and children in the hierarchy. We illustrate the effectiveness of the technique in the matching of line-segment groupings in SAR images of rural scenes.
\end{abstract}

\section{Introduction}

Marr [9] was one of the first to argue in a principled way for the hierarchical organisation and processing of information in vision systems. In practice the hierarchy may either be pyramidal [5], in which case the different levels represent different image resolutions, or it may be a conceptual hierarchy [7] in which case the different levels represent different degrees of abstraction from the available visual information. The key element in the development of techniques for hierarchical information processing is to exploit not only the intra-level constraints applying at the individual levels of representation but also inter-level constraints operating between different levels of the hierarchy. If used effectively these interlevel constraints can be brought to bear on the interpretation of uncertain image entities in such a way as to improve the fidelity of interpretation achieved by single level means. Viewed as an additional information source, inter-level constraints can be used to resolve ambiguities that would persist if single-level constraints alone were used.

Although hierarchical models clearly provide a powerful source of constraints for intermediate level scene interpretation, the available methodology for effectively and objectively utilising them is relatively restricted. In fact, the majority of the algorithms reported in the literature confine themselfs to low or intermediate level vision $[1,5]$. For instance Cohen et. al. have developed hierarchical Markov models for image segmentation [1]. Gidas [5], on the other hand, has used re-normalisation group ideas to improve the efficiency of simulated annealing for pyramidal image restoration using Markov-chains. As an example from intermediate-level vision, Henderson [7] has developed a form of syntactic 
discrete relaxation algorithm based upon a hierarchical shape process for $2 \mathrm{D}$ bject recognition. Drawing on a more expresive shape representation based on geons, Dickinson, Pentland and Rosenfeld [3] have matched aspect graphs using a Bayesian framework to represent hierarchical constraints. Sengupta and Boyer have developed a Bayesian hierarchical technique for indexing into large structural model-bases [11] using parametric relational descriptions. The technique has recently been rendered more efficient by the addition of geometric hashing [12].

Our aim in this paper is to offer a new addition to the literature by developing an iterative discrete relaxation algorithm for matching hierarchical relational models. Our starting point is to establish a Bayesian viewpoint for measuring the consistency of match for in a hierarchical manner by using both inter-level and intra-level constraints derived from a relational model. The approach builds on the framework that allowed Wilson and Hancock [13] to formulate single level relational matching as an iterative discrete relaxation process. It involves specifying the discrete relaxation scheme in terms of distinct probability distributions which dichotomise the roles of intra-level and inter-level constraint processes. The key elements of this specification are to define what we mean by a hierarchy and to stipulate how the information is propagated between the different levels of processing. Here we focus on conceptual hierarchies. This is a heterogeneous structure $[2,7,10]$ in which different label types and different classes of constraint operate at different levels of abstraction; this is to contrasted with the more familiar pyramidal hierarchy which is effectively homogeneous [1, 5]. Since we are dealing with discrete entities inter-level information communication is via a symbolic interpretation of the objects under consideration.

The outline of this paper is as follows: In section 2 we establish our Bayesian framework for discrete relaxation. Section 3 describes how the matching of a hierarchy can be mapped onto our discrete relaxation algorithm. Section 4 presents some experiments involving the matching of field boundaries in SAR images against digital map data. Finally Section 5 provides some conclusions.

\section{A Hierarchical Criterion}

We begin by establishing a simple formalism to describe hierarchically structured graphs. The hierarchical structure which we describe here is based on homogeneity of object-types within individual levels and heterogeneity between levels. That is to say that all objects at one level of the hierarchy have an identical relationship to the level above, the level below and to each other. In Section 4 we will consider an example from computer vision which involves the perceptual grouping of line tokens into corners. Here corners form a single level of the hierarchy, consisting of lines at the level below, and may be adjacent by the virtue of sharing a common line. One final limitation on the hierarchy is that all objects at one level are entirely constructed from units in the level below. In other words scene detail is subsumed at the higher levels, but no new features are introduced. 
The hierarchy consists of a number of levels, each containing objects which are fully described by their children at the level below. Formally each level is described by an attributed relational graph $G^{l}=\left(V^{l}, E^{l}, \mathbf{X}^{l}\right), \forall l \in L$, with $L$ being the index-set of levels in the hierarchy; $t$ and $b$ are used to denote the top and bottom levels of the hierarchy respectively. According to our notation for level $l$ of the hierarchy, $V^{l}$ is the set of nodes, $E^{l}$ is the set of intra-level edges and $\mathbf{X}^{l}=\left\{\underline{x}_{u}^{l}, \forall u \in V^{l}\right\}$ is a set of unary attributes residing on the nodes. The children or descendents which form the representation of an element $j$ at a lower level are denoted by $\mathcal{D}_{j}$. In other words, if $u^{l-1}$ is in $\mathcal{D}_{j}$ then there is a link in the hierarchy between element $j$ at level $l$ and element $u$ at level $l-1$. According to our assumptions, the elements of $\mathcal{D}_{j}$ are drawn exclusively from $V^{l-1}$.

The goal of performing relaxation operations is to find the match between scene graph $G_{1}$ and model graph $G_{2}$. This match is represented by a mapping function $f^{l}, \forall l \in L$ where $f^{l}: V_{1}^{l} \rightarrow V_{2}^{l}$.

The vertical structure that we consider in this paper is a subsumption hierarchy. In general, the upper levels of such a hierarchy are more sparsely populated with entities than the lower levels, due to the amalgamation of scene structure into higher-level relational units. The upper levels are more closely related to world structure while the lower levels represent raw image structure. It is for this reason that it is difficult to match the world-orientated upper levels using image information alone. Such a representational hierarchy is therefore necessary in order to propagate image information through increasingly more abstract representations.

The development of a hierarchical criterion proceeds along a similar line to the single-level relational consistency measure developed by Wilson and Hancock [13]. The quantity of interest is the MAP estimate for the mapping function $f$ given the available unary attributes, i.e. $P\left(f^{l}, \forall l \in L \mid \mathbf{X}^{l}, \forall l \in L\right)$. We factorise the measurement information over the set of nodes by application of Bayes rule under the assumption of measurement independence on the nodes. As a result

$$
P\left(f^{l}, \forall l \in L \mid \mathbf{X}^{l}, \forall l \in L\right)=\frac{1}{p\left(\mathbf{X}^{l}, \forall l \in L\right)}\left\{\prod_{l \in L} \prod_{u \in V^{l}} p\left(\mathbf{x}_{u}^{l} \mid f^{l}(u)\right)\right\} P\left(f^{l}, \forall l \in L\right)
$$

The critical modelling ingredient in developing a discrete relaxation scheme from the above MAP criterion is the joint prior for the mapping function, i.e. $P\left(f^{l}, \forall l \in L\right)$ which represents the influence of structural information on the matching process. The joint measurement density, $p\left(\mathbf{X}^{I}, \forall l \in L\right)$, on the other hand is a fixed property of the hierarchy and can be eliminated from further consideration.

The information provided to us from the scene is generally in terms of image primitives such as line segments or regions. This information is only directly relevant to the lowest level of the hierarchy in which the representation is closest to the image. Our task is therefore to propagate this information upwards through the hierarchy. To commence the formulation of a hierarchical matching scheme, we assume levels are conditionally dependent only on the levels directly above 
and below. This assumption allows the factorisation of the joint probability in a manner analogous to a Markov chain of probabilities [5]. Since we wish to draw information from the bottom upwards, the factorisation commences from the highest level of labelling. The expression for the joint probability of the hierarchical labelling is

$$
P\left(f^{l}, \forall l \in L\right)=P\left(f^{b}\right) \prod_{l \in L, l \neq t} P\left(f^{l+1} \mid f^{l}\right)
$$

We can now focus our attention on the conditional probabilities $P\left(f^{l+1} \mid f^{l}\right)$. These quantities express the probability of a labelling at the current level given a previously defined labelling at a lower level. We can use the concept of decomposing the graph into super-clique units to evaluate this probability in a similar fashion to that already exploited by Wilson and Hancock [13]. However in the hierarchical case the matching of child nodes is also important in gauging the quality of match.

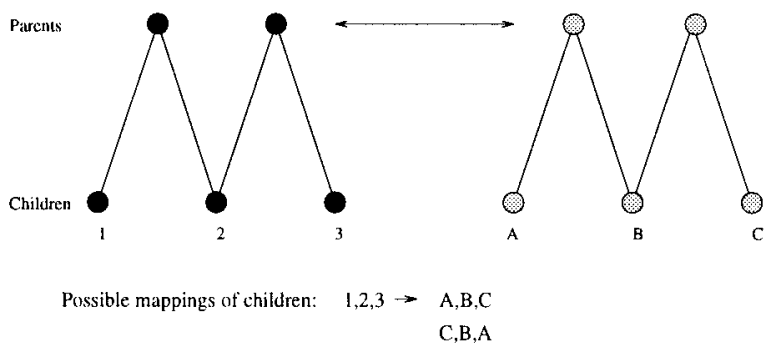

Fig. 1. Example constrained children mappings

The basic relational units that we use to describe graph structure are subgraphs consisting of the sets of nodes connected to a centre-node by an edge of the model-graph. We follow the same philosophy adopted by Wilson and Hancock [13] in the single-level case by assuming that the best estimate of the conditional probability being the mean value of the matching probabilities for these basic structural units or super-cliques. If $\Gamma_{j}^{l} \subset f^{l}$ denotes the current match of the super-clique centred on the object $j \in V_{1}^{l}$ then we write

$$
P\left(f^{l} \mid f^{l-1}\right)=\frac{1}{\left|V^{l}\right|} \sum_{j \in V^{l}} P\left(\Gamma_{j}^{l} \mid f^{l-1}\right)
$$

In order to gauge this probability, we require a dictionary of corresponding graph sub-units (super-cliques) from the model graph $G_{2}$. The allowed mappings between the model graph and the data graph which preserve the topological graph structure at a particular level of representation are referred to as "structure preserving mappings" or SPM's. It is important to note that we need only to explore only those mappings which are topologically identical to the super-clique 
$j$ and therefore the possible mappings of the child nodes are heavily constrained by the mappings of the parents (Figure 1). We denote the set of SPM's by $\mathcal{P}$. Since the set $\mathcal{P}$ is effectively the state-space of legal matching, we can apply the Bayes theorem to compute the conditional super-clique probability in the following manner

$$
P\left(\Gamma_{j}^{l} \mid f^{l-1}\right)=\sum_{S \in \mathcal{P}} P\left(\Gamma_{j}^{l} \mid S, f^{l-1}\right) P\left(S \mid f^{l-1}\right)
$$

We can now see that there are two distinct elements to our model. The first element is the comparison between our mapped realisation of the super-clique from graph $G_{1}, \Gamma_{j}^{l}$, with the selected unit from graph $G_{2}$ and the mapping from level $l-1$. Here we take the view that once we have hypothesised a particular mapping $S$ from $\mathcal{P}$, the mapping $f^{l-1}$ provides us with no further information. The matched super-clique $\Gamma_{j}^{l}$ is conditionally independent given a mapping from the set of SPM's and we may write the first term as $P\left(\Gamma_{j}^{l} \mid S\right)$. In other words, this first conditional probability models intra-level constraints. The second term is the significant one in evaluating the impact inter-level constraints on the labelling at the previous level - the possible mappings are weighted according to their probability given the mapping at the level below. The final expression for superclique matching probability is

$$
P\left(\Gamma_{j}^{l} \mid f^{l-1}\right)=\sum_{S \in \mathcal{P}} P\left(\Gamma_{j}^{l} \mid S\right) P\left(S \mid f^{l-1}\right)
$$

All that remains now is to evaluate these two probabilities according to the node labels they contain. Under the assumption of memoryless matching errors, the first term may be factorised over the marginal probabilities for the component labels $\gamma_{i}^{l}$ of the matched super-clique $\Gamma_{j}^{l}$ and their counterparts $s_{i}$ belonging to the structure preserving mapping $S$. In other words,

$$
P\left(\Gamma_{j}^{l} \mid S\right)=\prod_{\gamma_{i}^{l} \in \Gamma_{j}^{l}} P\left(\gamma_{i}^{l} \mid s_{i}\right)
$$

In order to proceed we need to specify a probability distribution for the different matching possibilities. There are three possible cases. Firstly, the match $\gamma_{i}^{l}$ may be to a dummy-node $d$ inserted into $\Gamma_{j}^{l}$ to raise it to the same size as $S$ so as to facilitate comparison. The second and third cases, relate to whether the match is correct or in error. Assuming that dummy node insertions may be made with probability $P_{s}$ and that matching errors occur with probability $P_{e}$, then we can write down the following distribution rule

$$
P\left(\gamma_{i}^{l} \mid s_{i}\right)= \begin{cases}P_{s} & \text { if } \gamma_{i}^{l}=d \text { or } s_{i}=d \\ \left(1-P_{e}\right)\left(1-P_{s}\right) & \text { if } \gamma_{i}^{l}=s_{i} \\ P_{e}\left(1-P_{s}\right) & \text { otherwise }\end{cases}
$$

The second term in Equation (5) is more subtle; it represents the conditional probability of the SPM $S$ given a previously determined labelling at the level 
below. However, the mapping contains labels only from the current level $l$, not labels from level $l-1$. We can reconcile this difference by noting that selection of a particular mapping at level $l$ limits the number of consistent mappings allowed topologically at the level below. In other words if one node is mapped to another at level $l$, the consistent interpretation is that the children of the nodes must match to each other. By applying this constraint, the labelling at $l-1$ and a set of allowed mappings of the child nodes can be used to gauge the probability of a particular SPM occuring. These legitimate mappings are referred to as "Hierarchy Preserving Mappings" or HPM's. It is these symbolic mappings that form the critical ingredient in our matching scheme and distinguish it from the parametric alternatives reported in the literature [11,12]. We will denote the set of HPM's derived from an SPM $S$ as $\mathcal{Q}_{S}$ and a member of this set as $Q=\left\{q_{i}, \forall i \in \mathcal{D}_{j}\right\}$. Using this model the conditional probability $P\left(S \mid f^{l-1}\right)$ is given by

$$
P\left(S \mid f^{l-1}\right)=\sum_{Q \in \mathcal{Q}_{S}} P\left(S \mid Q, f^{l-1}\right) P\left(Q \mid f^{l-1}\right)
$$

We can now assume that $S$ is conditionally independent of $f^{l-1}$ given $Q$, and arrive at the expression

$$
P\left(S \mid f^{l-1}\right)=\sum_{Q \in \mathcal{Q}_{S}} P(S \mid Q) P\left(Q \mid f^{l-1}\right)
$$

Traditionally, dictionary based hierarchical schemes have operated by using a labelling determined at a preceding level to reduce the dictionary set by elimination of vertically inconsistent items. This approach can easily be incorporated into our scheme by setting $P\left(Q \mid f^{l-1}\right)$ equal to 1 for consistent items and 0 for those which are inconsistent. However we propose a different approach; by adopting the same kind of label distribution used in Equation 7 we can grade the SPM's according to their consistency with the match at level $l-1$, i.e. $f^{l-1}$. The model is developed by factorising over the child nodes $q_{i} \in Q$ in the following manner

$$
P\left(Q \mid f^{l-1}\right)=\prod_{q_{i} \in Q} P\left(q_{i} \mid \gamma_{i}^{l-1}\right)
$$

The conditional probabilities are assigned by a re-application of the distribution rule given in Equation (7), i.e.

$$
P\left(q_{i} \mid f^{l-1}\right)= \begin{cases}P_{s} & \text { if dummy node match } \\ \left(1-P_{e}^{l-1}\right)\left(1-P_{s}\right) & \text { if } q_{i}=\gamma_{i}^{l-1} \\ P_{e}^{l-1}\left(1-P_{s}\right) & \text { otherwise }\end{cases}
$$

In this case, however, the value of $P_{e}^{l-1}$ must be set to reflect the prevailing level of label-errors at level $l-1$. For the conditional probability of the SPM given the HPM $Q$, we adopt a simple uniform model under the assumption that all legitimate mappings are equivalent, i.e. $P(S \mid Q)=P(S)=\frac{1}{|\mathcal{P}|}$. 


\section{Discrete Relaxation With Hierarchical Corner Graphs}

In this section we develop a hierarchical matching scheme based on line-segments and corner objects. The method of extracting these elements from the scene is explained in detail in [13]. It commences by extracting straight-line segments from labelled feature points such as intensity edges or ridges. The extracted linear segments are then organised into meaningful perceptual units consisting of the corner groupings of interest here. The final element to the graph representation is a set of intra-level relationships. We establish the required neighbourhood relations by seeding a Voronoi tesselations from the raw line-tokens and computing the associated Delaunay; the edges of the Delaunay graph represent the intra-level graph constraints.

\subsection{Mappings}

The structure-preserving mappings (SPM's) represent intra-level structural information elicited from a Delaunay graph. The corresponding SPM's are generated in exactly the same fashion as described in [13]. The hierarchy-preserving mappings are generated in this specific corner/line representation by exploring the possible mapping of child lines given the corner match above. Since each corner consists of two line-segments, there are two HPM's for each corner participating in the SPM at the level above and hence $2|\mathcal{P}|$ HPM's altogether.

As described earlier we can explore the hierarchy in either direction. When descending the hierarchy corner mappings become the children of lines. In this case the HPM's are determined as follows: A super-clique of the lines consists of a central line and the external lines which are direct Voronoi neighbours of the centre. Consider the central line and one of the external lines; if they do not mutually participate in a corner relation there is no hierarchical constraint and the hierarchical portion of probability is ignored. If on the other hand they do participate in a corner, this unit can provide a hierarchical constraint. In this case there is just one HPM; the corner must map to the corresponding corner of the mapping of the two lines (see Figure 2).

Armed with these SPM's and HPM's we can evaluate the hierarchical criterion in Equation 3. The strategy we use is to first match the lines with a non-hierarchical criterion to obtain an initial labelling. Then we match the corner level with the hierarchical criterion incorporating information from the initial line labelling. Finally the lines are re-matched with the hierarchical criterion for traversing the hierarchical graph.

\section{Experimental Results and Discussion}

The discussion of the performance of the hierarchical scheme begins with a small test case to demonstrate how the hierarchical criterion can reduce ambiguity between similar graphs by introducing information from an additional level of representation. Figure 3 shows a graph which is symmetrical at the top level of 


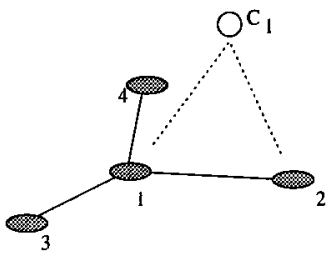

$\mathrm{G}_{1}$

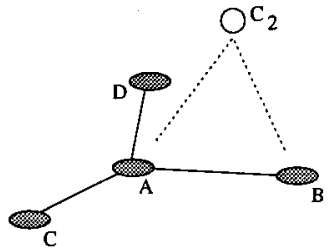

$\mathrm{G}_{2}$

$1->A_{\text {and }} 2->\mathrm{B}_{\text {implies }} \mathrm{C}_{\mathrm{P}}>\mathrm{C}_{2}$

Fig. 2. Example of an upwards HPM

representation and consequently ambiguous at that level. However at the next level there are definite differences between the children.

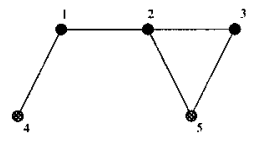

Dalla Grinh

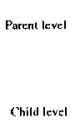

Child level

Fig. 3. Test case: Ambiguous graphs

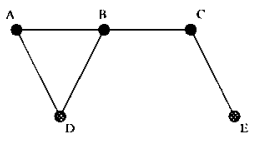

Model Griph

The graphs under consideration are ambiguous at the parent level because there is a symmetry which does not permit distinction between the mappings $(1,2,3) \rightarrow(A, B, C)$ and $(1,2,3) \rightarrow(C, B, A)$. Table 1 shows how the HPM's introduce information from the child level which distinguishes between the two possibilities.

\begin{tabular}{||c|c|c|c||}
\hline$(1,2,3) \rightarrow(A, B, C)$ HPM's & Value & $(1,2,3) \rightarrow(C, B, A)$ HPM's & Value \\
\hline \hline $5 \rightarrow D$ & $\mathrm{c}$ & $5 \rightarrow$ dummy & $\mathrm{b}$ \\
\hline $4 \rightarrow E$ & $\mathrm{c}$ & $d u m m y \rightarrow D$ & $\mathrm{~b}$ \\
\hline \hline & & $4 \rightarrow$ dummy & $\mathrm{b}$ \\
\hline & & dummy $E$ & $\mathrm{~b}$ \\
\hline \hline Total & $c^{2}$ & Total & $2 b^{2}$ \\
\hline
\end{tabular}

Table 1. Mappings between the test graphs: $c$ and $b$ represent the probabilities of matching errors

However this simple case does not address some important properties of real hierarchical graphs; segmentation of real images into a hierarchical structure is difficult and prone to error. For this reason the method has also been tested on 
some real data; the data under study here is based on the SAR data discussed in [13] and consists of linear field boundaries at the lowest level and corners and the top level. In this example the matching application involves finding correspondences between line segments extracted from the SAR data and their counterparts in a digital map. We have extracted from the SAR only those line segments for which a feasible match exists in the map model. In other words, all lines in the data have a matching line in the model. Corruption has then been added by deleting a certain number of lines in the data and reinserting an identical number of lines at random positions and orientations. Figure 4 contains an example of such a process.

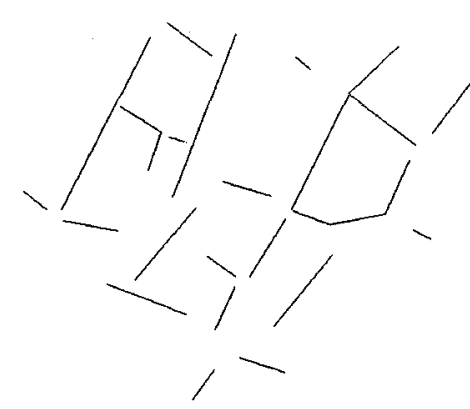

Corrupted Data

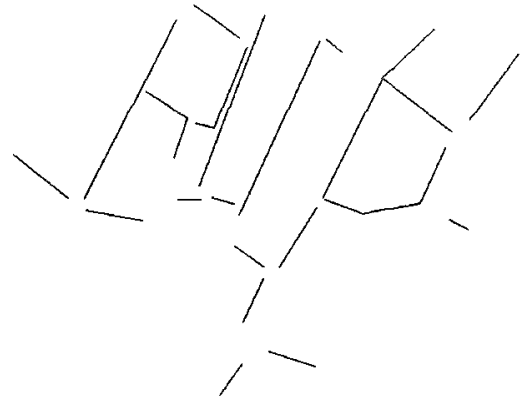

Original Data

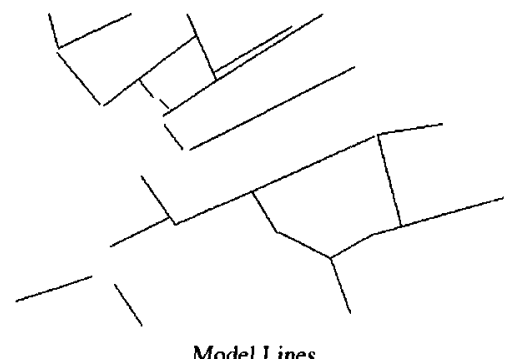

Fig. 4. Example hierarchical datasets

Table 2 provides a summary of the performance of the hierarchical matching of lines and corners. The performance is summarised in terms of the fraction of line segments matched correctly $F_{c}$.

\section{Conclusions}

We have developed graph matching technique which is tailored to hierarchical relational descriptions. The key element is this development is to quantify the matching consistency using the concept of hierarchy preserving mappings between two graphs. Central to the development of this novel technique is the 


\begin{tabular}{||c|c||}
\hline Level of Corruption & Hierarchical relaxation $F_{c}$ \\
\hline \hline 0.15 & 0.45 \\
\hline 0.2 & 0.56 \\
\hline 0.27 & 0.47 \\
\hline 0.34 & 0.42 \\
\hline
\end{tabular}

Table 2. Results of hierarchical relaxation

idea of computing the probability of a particular node match by drawing on the topologically allowed mappings of the child nodes in the hierarchy. Examination of a test case has shown that this method can disambiguate graphs which are ambiguous at one level of abstraction. Results on image data with lines and corners as graph nodes reveal that the technique is capable of matching perceptual groupings under moderate levels of corruption.

\section{References}

1. F. Cohen and D. Cooper. Simple Parallel Hierarchical and Relaxation Algorithms for Segmenting Non-Causal Markovian Random Fields. IEEE PAMI, 9, 1987, pp. 195-219.

2. L. Davis and T. Henderson. Hierarchical Constraint Processes for Shape Analysis. IEEE PAMI, 3, 1981, pp.265-277.

3. S.J. Dickinson, A.P.Pentland and A. Rosenfeld. 3-D Shape Recovery using Distributed Aspect Matching. IEEE PAMI, 14, 1992, pp. 174-198.

4. S.J. Dickinson, A.P.Pentland and A. Rosenfeld. From volumes to views: An approach to $3 D$ object recognition. CVGIP: Image Understanding, 55, 1992, pp. $130-154$.

5. B. Gidas. A Renormalization Group Approach to Image Processing Problems. IEEE PAMI, 11, 1989, pp.164-180.

6. E. R. Hancock and J. Kittler, Discrete Relaxation. Pattern Recognition, 23, 1990, pp.711-733.

7. T. Henderson. Discrete Relaxation Techniques. Oxford University Press, 1990.

8. J. Liang, H.I. Chrstensen and F.V. Jensen, Qualitative Recognition using Bayesian Reasoning. Pattern Recognition in Progress IV, Edited by E.S Gelsema and L.N Kanal, 1994, pp. 255-266.

9. D. Marr, Vision. W.H. Freeman and Co., San Francisco.

10. J. Qian and R. Ehrich. A Framework for Uncertainty Reasoning in Hierarchical Visual Evidence Space. Proceedings of the $10^{\text {th }}$ International Conference on Pattern Recognition, Vol. 1, 1990, p.119-124.

11. K. Sengupta and K.L. Boyer, Organising Large Structural Model-bases. IEEE PAMI, 17, 1995, pp. 321-332.

12. K. Sengupta and K.L. Boyer, Using Geometric Hashing and Information Theoretic Clustering for Fast Recognition from Large CAD Modelbases. IEEE International Symposium on Computer Vision, 1995, pp. 151-156.

13. R.C. Wilson, A.N. Evans and E. R. Hancock, Relational Matching by Discrete Relaxation. Image and Vision Computing, 13, 1995, pp. 411-422. 